\title{
FORTIMICIN A PRODUCTION BY MICROMONOSPORA OLIVOASTEROSPORA IN A CHEMICALLY DEFINED MEDIUM
}

\author{
Mitsuyoshi Yamamoto, Ryo OKachi, Isao Kawamoto and Takashi Nara \\ Tokyo Research Laboratory, Kyowa Hakko Kogyo Co., Ltd. \\ 3-6-6 Asahimachi, Machidashi, Tokyo, Japan \\ (Received for publication October 1, 1977)
}

\begin{abstract}
A chemically defined medium was devised in order to study the requirements for fortimicin A production by Micromonospora olivoasterospora KY 11515. Soluble starch was the best carbon source; $\mathrm{NH}_{4} \mathrm{Cl}$ and $\mathrm{NH}_{4} \mathrm{NO}_{3}$ were suitable nitrogen sources both for the growth and fortimicin production. Amino acids such as L-arginine, L-asparagine, L-aspartic acid and L-glutamic acid showed some stimulatory effects on both growth and antibiotic production of M. olivoasterospora while L-serine stimulated only antibiotic production and L-citrulline only the growth. $\mathrm{K}_{2} \mathrm{HPO}_{4}, \mathrm{MgSO}_{4} \cdot 7 \mathrm{H}_{2} \mathrm{O}$ and $\mathrm{CaCO}_{3}$ were essential especially for the antibiotic production. The most important finding was that vitamin $\mathrm{B}_{12}$, cobalt and nickel showed marked stimulatory effects on fortimicin A production.
\end{abstract}

Micromonospora olivoasterospora produced fortimicin A, fortimicin B and some other minor components in the fermentation broth ${ }^{1 \sim 4}$. Fortimicins $\mathrm{A}$ and $\mathrm{B}$ were isolated as purified materials ${ }^{2)}$ and the structural studies revealed that fortimicin A was a unique aminocyclitol-aminoglycoside antibiotic ${ }^{5)}$ with broad antibacterial activities ${ }^{1,6)}$. Fortimicins $\mathrm{A}$ and $\mathrm{B}$ are pseudodisaccharides and incorporate a novel aminocyclitol fortamine, which is a 1,4-diamine, contains both $\mathrm{N}$ - and O-methyl groups and has an unusual stereochemistry as shown in Fig. 1.

Fig. 1. Structures of fortimicins A and B
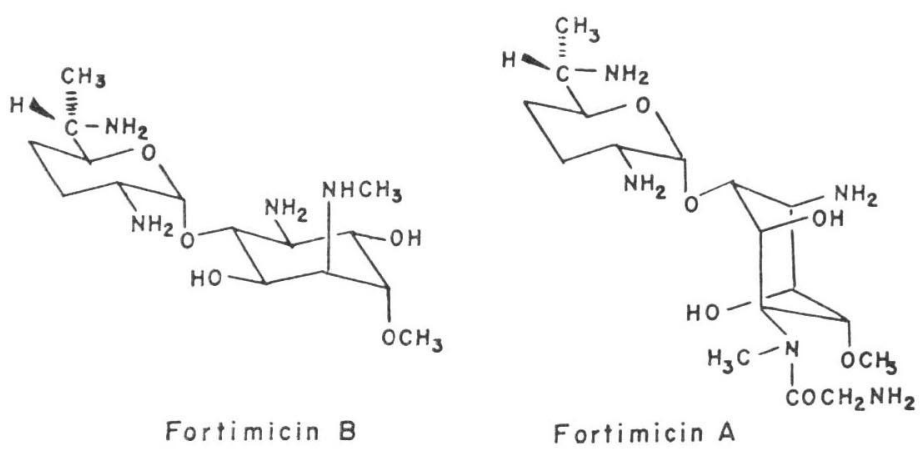

Micromonospora have been reported to produce several antibiotics, but no report has appeared on antibiotic production in a chemically defined medium except for gentamicin production in a synthetic medium reported by TeSTA et al..$^{i)}$ Development of a chemically defined medium for $M$. olivoasterospora was investigated to determine the factors affecting the growth and/or production of fortimicins. 


\section{Materials and Methods}

\section{Producing Organism}

The organism used in this investigation was a high productivity mutant, Micromonospora olivoasterospora KY 11515, induced from the parent M. olivoasterospora MK-70, ATCC 21819. The mutant had physiologically and morphologically similar characteristics to the parent strain. The culture was maintained in lyophile tubes and each fermentation grown at $30^{\circ} \mathrm{C} 2 \sim 3$ weeks on an agar slant consisting of $0.4 \%$ yeast extract, $1.0 \%$ malt extract, $0.4 \%$ glucose and $2.0 \%$ agar, pH 7.2 .

Fermentation

All fermentations were carried out in 300-ml Erlenmeyer flasks containing 30-ml of fermentation medium. Flasks were shaken at $30^{\circ} \mathrm{C}$ on a rotary shaker rotating within a $5-\mathrm{cm}$ circle at $190 \mathrm{rpm}$, usually for $4 \sim 6$ days. Flasks were sampled daily for assay. If fermentation time is not indicated in a table the data show maximum antibiotic potencies during that experiment. For the experiments on carbon sources and inorganic nitrogen sources, three times washed inoculum from a complex organic medium was transferred to the fermentation medium. The complex organic medium consisted of $1 \%$ soluble starch, $1 \%$ glucose, $0.1 \%$ yeast extract, $0.5 \%$ peptone and $0.1 \% \mathrm{CaCO}_{3}$. For all other experiments, three times washed inoculum from slants was transferred to the $50 \mathrm{ml}$ of seed medium in a $300-\mathrm{ml}$ Erlenmeyer flask, which was cultivated at $30^{\circ} \mathrm{C}$ for 4 days on a rotary shaker. The seed medium consisted of $2 \%$ soluble starch, $0.5 \%$ glucose, $0.5 \% \mathrm{NH}_{4} \mathrm{Cl}, 0.05 \% \mathrm{~K}_{2} \mathrm{HPO}_{4}, 0.05 \% \mathrm{MgSO}_{4} \cdot 7 \mathrm{H}_{2} \mathrm{O}$ and $0.1 \% \mathrm{CaCO}_{3}$. Contents from several seed flasks were then mixed. Ten percent inoculum was transferred to the fermentation medium. All media used in this investigation were adjusted to $\mathrm{pH}$ 7.2 with $\mathrm{HCl}$ or $\mathrm{NaOH}$ and then sterilized by autoclaving at $120^{\circ} \mathrm{C}$ for 15 minutes.

Determination of Growth

The method reported by WaGMan et al. ${ }^{8)}$ was used.

Preparation of Samples for Antibiotic Assay

Four tenths $\mathrm{ml}$ of $6 \mathrm{~N} \mathrm{HCl}$ was added to $10 \mathrm{ml}$ of fermented broth, which was then stirred thoroughly to release fortimicins bound to the mycelia and finally centrifuged. The supernatant was adjusted to pH 8 with $6 \mathrm{~N} \mathrm{NH}_{4} \mathrm{OH}$ or diluted by 20 times with $0.1 \mathrm{M}$ Tris- $\mathrm{HCl}$ buffer, $\mathrm{pH} 8$, for bioassay. For the chemical assay, the intact acidified supernatant was used.

\section{Chemical Assay of Fortimicins}

For chemical assay of each component of fortimicins, a modified method of Benjamin et al. ${ }^{9 \text { ) }}$ was used. Samples were applied to silica-gel plates which were then developed with lower phase of chloroform - methanol - ammonia (1:1:1). The plate was then developed in a solution of 7-chloro-4nitrobenzo-2-oxa-1,3-diazole (NBD chloride, E. Merck \& Co.) in acetone $(2 \mathrm{mg} / \mathrm{ml})$. The plate was heated at $120^{\circ} \mathrm{C}$ for 10 minutes, and then rechromatographed in acetone, repeatedly. Fluorescent spots were measured by a fluorometer (Shimazu CS-900) and the fortimicins were calculated by a standard curve. Details of the chemical assay of fortimicins will be reported elsewhere.

\section{Bioassay for Total Antibiotic Potency}

An agar diffusion assay using Bacillus subtilis KY 4273 was used. Potency was expressed in $\mu \mathrm{g} / \mathrm{ml}$ of a standard fortimicin A sulfate of $537 \mu \mathrm{g} / \mathrm{mg}$.

\section{Results}

\section{Effects of Inorganic Nitrogen Sources}

Effects of several kinds of inorganic nitrogen compounds on the growth and antibiotic production were studied in a basal fermentation medium containing $2 \%$ soluble starch, $0.5 \%$ glucose, $0.05 \%$ $\mathrm{K}_{2} \mathrm{HPO}_{4}, 0.05 \% \mathrm{MgSO}_{4} \cdot 7 \mathrm{H}_{2} \mathrm{O}$ and $0.1 \% \mathrm{CaCO}_{3}$ (Table 1). $\mathrm{NH}_{4} \mathrm{Cl}, \mathrm{NH}_{4} \mathrm{NO}_{3}$ and $\left(\mathrm{NH}_{4}\right)_{2} \mathrm{SO}_{4}$ increased growth slightly more than $\mathrm{KNO}_{3}$ and $\mathrm{NaNO}_{3}$. This organism could not utilize $\mathrm{NaNO}_{2}$. On the other hand, ammonium ion increased antibiotic production more significantly than nitrate or nitrite. $\mathrm{NH}_{4} \mathrm{Cl}$ 
Table 1. Effects of inorganic nitrogen sources

\begin{tabular}{|c|c|c|c|c|c|c|c|}
\hline \multirow{2}{*}{\multicolumn{2}{|c|}{$\begin{array}{c}\text { Inorganic nitrogen } \\
\text { source (M) }\end{array}$}} & \multicolumn{3}{|c|}{4 days } & \multicolumn{3}{|c|}{7 days } \\
\hline & & \multirow{2}{*}{$\begin{array}{c}\mathrm{pH} \\
5.5 \\
5.4\end{array}$} & \multirow{2}{*}{$\begin{array}{c}\begin{array}{c}\text { Growth } \\
(\mathrm{mg} / \mathrm{ml})\end{array} \\
4.25 \\
3.39\end{array}$} & \multirow{2}{*}{$\begin{array}{c}\text { Antibiotics } \\
(\mu \mathrm{g} / \mathrm{ml})\end{array}$} & \multirow{2}{*}{$\begin{array}{l}\mathrm{pH} \\
4.4 \\
3.3\end{array}$} & \multirow{2}{*}{$\begin{array}{c}\begin{array}{c}\text { Growth } \\
(\mathrm{mg} / \mathrm{ml})\end{array} \\
3.40 \\
2.57\end{array}$} & \multirow{2}{*}{$\begin{array}{c}\begin{array}{c}\text { Antibiotics } \\
(\mu \mathrm{g} / \mathrm{ml})\end{array} \\
5 \\
4\end{array}$} \\
\hline $\mathrm{NH}_{4} \mathrm{Cl}$ & $\begin{array}{l}0.1 \\
0.05\end{array}$ & & & & & & \\
\hline$\left(\mathrm{NH}_{4}\right)_{2} \mathrm{SO}_{4}$ & $\begin{array}{l}0.1 \\
0.05\end{array}$ & $\begin{array}{l}5.8 \\
5.6\end{array}$ & $\begin{array}{l}5.09 \\
4.24\end{array}$ & $\begin{array}{l}3 \\
4\end{array}$ & $\begin{array}{l}4.5 \\
4.6\end{array}$ & $\begin{array}{l}4.26 \\
3.38\end{array}$ & $\begin{array}{r}<1 \\
3\end{array}$ \\
\hline $\mathrm{NH}_{4} \mathrm{NO}_{3}$ & $\begin{array}{l}0.1 \\
0.05\end{array}$ & $\begin{array}{l}5.5 \\
5.4\end{array}$ & $\begin{array}{l}3.41 \\
3.38\end{array}$ & $\begin{array}{l}4 \\
5\end{array}$ & $\begin{array}{l}4.7 \\
4.8\end{array}$ & $\begin{array}{l}3.37 \\
3.40\end{array}$ & $\begin{array}{l}4 \\
6\end{array}$ \\
\hline $\mathrm{KNO}_{3}$ & $\begin{array}{l}0.1 \\
0.05\end{array}$ & $\begin{array}{l}7.3 \\
7.5\end{array}$ & $\begin{array}{l}3.29 \\
2.54\end{array}$ & $\begin{array}{l}<1 \\
<1\end{array}$ & $\begin{array}{l}8.5 \\
8.4\end{array}$ & $\begin{array}{l}2.57 \\
2.53\end{array}$ & $\begin{array}{l}<1 \\
<1\end{array}$ \\
\hline $\mathrm{NaNO}_{3}$ & $\begin{array}{l}0.1 \\
0.05\end{array}$ & $\begin{array}{l}7.3 \\
7.6\end{array}$ & $\begin{array}{l}2.49 \\
2.60\end{array}$ & $\begin{array}{l}<1 \\
<1\end{array}$ & $\begin{array}{l}8.3 \\
8.4\end{array}$ & $\begin{array}{l}4.28 \\
2.55\end{array}$ & $\begin{array}{l}<1 \\
<1\end{array}$ \\
\hline $\mathrm{NaNO}_{2}$ & $\begin{array}{l}0.1 \\
0.05\end{array}$ & $\begin{array}{l}6.8 \\
6.8\end{array}$ & $\begin{array}{l}0.00 \\
0.00\end{array}$ & $\begin{array}{l}<1 \\
<1\end{array}$ & $\begin{array}{l}7.0 \\
7.0\end{array}$ & $\begin{array}{l}0.00 \\
0.00\end{array}$ & $\begin{array}{l}<1 \\
<1\end{array}$ \\
\hline none & & 6.7 & 0.00 & $<1$ & 7.0 & 0.00 & $<1$ \\
\hline
\end{tabular}

Basal fermentation medium: soluble starch $2 \%$, glucose $0.5 \%, \mathrm{~K}_{2} \mathrm{HPO}_{4} 0.05 \%, \mathrm{MgSO}_{4} \cdot 7 \mathrm{H}_{2} \mathrm{O} 0.05 \%$, $\mathrm{CaCO}_{3} 0.1 \%$.

Table 2. Effects of carbon sources

\begin{tabular}{l|c|c|c|l|c|c|c}
\hline \multicolumn{1}{c}{$\begin{array}{c}\text { Carbon } \\
\text { source }\end{array}$} & $\mathrm{pH}$ & $\begin{array}{c}\text { Growth } \\
(\mathrm{mg} / \mathrm{ml})\end{array}$ & $\begin{array}{c}\text { Antibiotics } \\
(\mu \mathrm{g} / \mathrm{ml})\end{array}$ & $\begin{array}{c}\text { Carbon } \\
\text { source }\end{array}$ & $\mathrm{pH}$ & $\begin{array}{c}\text { Growth } \\
(\mathrm{mg} / \mathrm{ml})\end{array}$ & $\begin{array}{c}\text { Antibiotics } \\
(\mu \mathrm{g} / \mathrm{ml})\end{array}$ \\
\hline Soluble starch & 4.9 & 3.38 & 3 & D-Mannose & 6.0 & $\operatorname{tr}$ & $<1$ \\
D-Raffinose & 6.8 & $\operatorname{tr} *$ & $<1$ & D-Xylose & 6.0 & $\operatorname{tr}$ & $<1$ \\
Maltose & 5.7 & 3.25 & 2 & D-Arabinose & 6.1 & $\operatorname{tr}$ & $<1$ \\
Sucrose & 6.4 & 0.85 & $<1$ & Glycerine & 6.8 & $\operatorname{tr}$ & $<1$ \\
Lactose & 6.5 & $\operatorname{tr}$ & $<1$ & Sorbitol & 6.9 & $\operatorname{tr}$ & $<1$ \\
D-Galactose & 6.3 & $\operatorname{tr}$ & $<1$ & Mannitol & 6.9 & $\operatorname{tr}$ & $<1$ \\
Glucose & 5.3 & 1.75 & $<1$ & Inositol & 6.9 & $\operatorname{tr}$ & $<1$ \\
L-Rhamnose & 6.4 & $\operatorname{tr}$ & $<1$ & None & 6.8 & $\operatorname{tr}$ & $<1$ \\
D-Fructose & 6.2 & $\operatorname{tr}$ & $<1$ & & & & $<$ \\
\hline
\end{tabular}

Basal fermentation medium: $\mathrm{NH}_{4} \mathrm{Cl} 0.5 \%, \mathrm{~K}_{2} \mathrm{HPO}_{4} 0.05 \%, \mathrm{MgSO}_{4} \cdot 7 \mathrm{H}_{2} \mathrm{O} 0.05 \%, \mathrm{CaCO}_{3} 0.1 \%$. $*$ tr: $\operatorname{trace}$ Each carbon source was added $4 \%$ in a basal medium.

and $\mathrm{NH}_{4} \mathrm{NO}_{3}$ were slightly better for increasing fortimicin production than $\left(\mathrm{NH}_{4}\right)_{2} \mathrm{SO}_{4}$.

\section{Effects of Carbon Sources}

The effects of carbohydrates on the growth and antibiotic production shown in Table 2 were studied in a fermentation medium containing $4 \%$ carbohydrate, $0.5 \% \mathrm{NH}_{4} \mathrm{Cl}, 0.05 \% \mathrm{~K}_{2} \mathrm{HPO}_{4}, 0.05 \% \mathrm{MgSO}_{4}$. $7 \mathrm{H}_{2} \mathrm{O}$ and $0.1 \% \mathrm{CaCO}_{3}$. Soluble starch, maltose, sucrose and glucose showed stimulatory effects on the growth. Other carbohydrates were not utilized by the organism. Organic acids such as sodium citrate, sodium fumarate and sodium acetate also failed to support growth. Among the four carbohydrates which stimulated the growth, only soluble starch and maltose produced antibiotic activity in the broth. When $0.5 \%$ of L-arginine was added to the fermentation medium, the effect of soluble starch was distinguished more clearly from the other carbohydrates by both growth and antibiotic production.

Effects of Amino Acids

The effects of more than 20 amino acids were tested in a basal fermentation medium containing 
$4 \%$ soluble starch, $0.5 \% \mathrm{NH}_{4} \mathrm{Cl}, 0.05 \% \mathrm{~K}_{2} \mathrm{HPO}_{4}$, $0.05 \% \mathrm{MgSO}_{4} \cdot 7 \mathrm{H}_{2} \mathrm{O}, 0.1 \% \mathrm{NaCl}$ and $0.1 \% \mathrm{Ca}-$ $\mathrm{CO}_{3}$. Among them, L-arginine, L-asparagin, L-aspartic acid and L-glutamic acid showed stimulatory effects both on the growth and antibiotic production. L-Citrulline stimulated only growth while L-serine increased only fortimicin production. The data on L-arginine, L-serine, and L-asparagine are shown in Table 3. Many combinations of these amino acids were tested but no further improvement was found.

Fig. 2. Combination effects of mannitol with soluble starch

Basal fermentation medium: soluble starch $4 \%$, $\mathrm{NH}_{4} \mathrm{Cl} 0.5 \%$, L-arginine $0.5 \%, \mathrm{~K}_{2} \mathrm{HPO}_{4} \quad 0.05 \%$, $\mathrm{MgSO}_{4} \cdot 7 \mathrm{H}_{2} \mathrm{O} 0.05 \%, \mathrm{CaCO}_{3} 0.1 \%$

Fortimicins were assayed by bioassay.

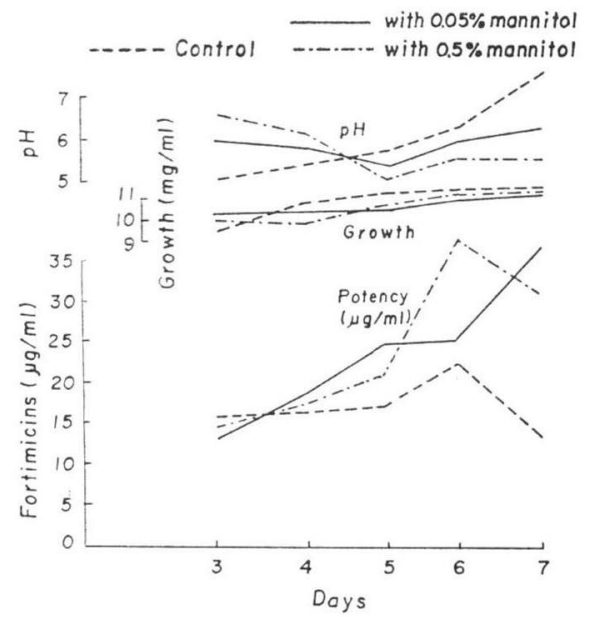

Table 3. Effects of amino acids

\begin{tabular}{l|c|c|c}
\hline \multicolumn{1}{c|}{ Amino acid } & $\mathrm{pH}$ & $\begin{array}{c}\text { Growth } \\
(\mathrm{mg} / \mathrm{ml})\end{array}$ & $\begin{array}{c}\text { Antibiotics } \\
(\mu \mathrm{g} / \mathrm{ml})\end{array}$ \\
\hline $0.5 \%$ L-Arginine & 5.1 & 9.40 & 34 \\
$0.2 \%$ L-Serine & 5.3 & 4.23 & 30 \\
$\begin{array}{l}1.0 \% \text { L- } \\
\quad \text { Asparagine }\end{array}$ & 6.9 & 9.33 & 32 \\
None & 5.1 & 4.22 & 24 \\
\hline
\end{tabular}

Basal fermentation medium: soluble starch $4 \%$, $\mathrm{NH}_{4} \mathrm{Cl} 0.5 \%, \mathrm{~K}_{2} \mathrm{HPO}_{4} 0.05 \%, \mathrm{MgSO}_{4} \cdot 7 \mathrm{H}_{2} \mathrm{O} 0.05$ $\%, \mathrm{NaCl} 0.1 \%, \mathrm{CaCO}_{3} 0.1 \%$

Fig. 3. Effects of $\mathrm{Ni}^{++}$in the presence of $\mathrm{Co}^{++}$on fortimicin production

Basal fermentation medium: soluble starch $4 \%$, mannitol $0.05 \%, \mathrm{NH}_{4} \mathrm{Cl} 0.5 \%$, L-arginine $0.5 \%$, $\mathrm{K}_{2} \mathrm{HPO}_{4} 0.05 \%, \mathrm{MgSO}_{4} \cdot 7 \mathrm{H}_{2} \mathrm{O} 0.1 \%, \mathrm{NaCl} 0.1 \%$, $\mathrm{CaCO}_{3} 0.1 \%, \mathrm{Co}^{++} 1 \times 10^{-4} \%$

Fortimicins $\mathrm{A}$ and $\mathrm{B}$ were assayed by chemical assay. $\mathrm{Co}^{++}$and $\mathrm{Ni}^{++}$were added as $\mathrm{CoCl}_{2} \cdot 6 \mathrm{H}_{2} \mathrm{O}$ and $\mathrm{NiCl}_{2} \cdot 6 \mathrm{H}_{2} \mathrm{O}$, respectively.

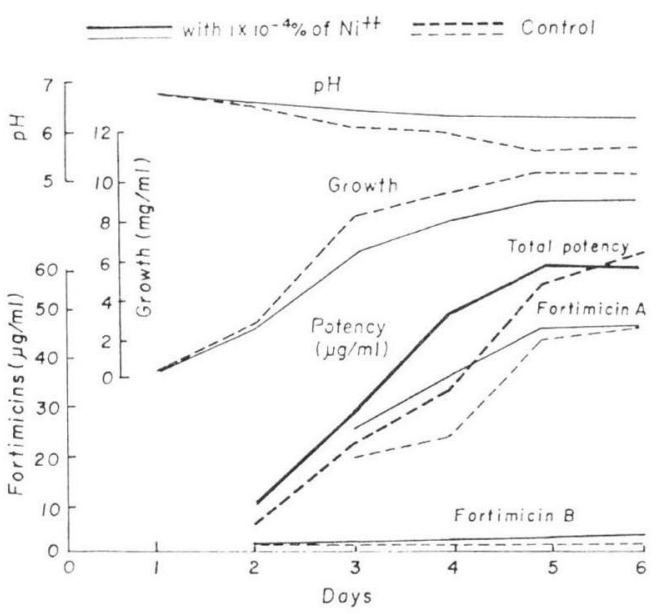

Combination Effects of Sugars with Soluble Starch

Maltose, sucrose, mannose, xylose, sorbitol, mannitol and glucose were added to a fermentation medium containing $4 \%$ soluble starch, $0.5 \% \mathrm{NH}_{4} \mathrm{Cl}, 0.5 \%$ L-arginine, $0.05 \% \mathrm{~K}_{2} \mathrm{HPO}_{4}, 0.05 \% \mathrm{MgSO}_{4}$. $7 \mathrm{H}_{2} \mathrm{O}$, and $0.1 \% \mathrm{CaCO}_{3}$ and their combination effects with soluble starch were examined.

None showed any effects on the growth, while mannitol and mannose showed almost the same effect on antibiotic production.

The combination effects of mannitol with soluble starch are shown in Fig. 2. The growth levels in the three conditions were the same. Whereas the broth $\mathrm{pH}$ of the control condition rose above $\mathrm{pH} 7$, the $\mathrm{pH}$ of the $0.05 \%$ or $0.5 \%$ mannitol-containing media remained below $\mathrm{pH} 6$ at the 7 th day. In the control medium, the antibiotic yield increased more slowly and decreased suddenly at the 7 th day. In the mannitol-containing media, fortimicin increased more rapidly to a $75 \%$ higher maximum and 
Table 4. Effects of inorganic salts

\begin{tabular}{|c|c|c|c|c|c|c|c|}
\hline$\underset{(\%)}{\mathrm{K}_{2} \mathrm{HPO}_{4}}$ & $\mathrm{pH}$ & $\begin{array}{l}\text { Growth } \\
(\mathrm{mg} / \mathrm{ml})\end{array}$ & $\begin{array}{c}\text { Antibiotics } \\
(\mu \mathrm{g} / \mathrm{ml})\end{array}$ & $\underset{(\%)}{\mathrm{MgSO}_{4} \cdot 7 \mathrm{H}_{2} \mathrm{O}}$ & $\mathrm{pH}$ & $\begin{array}{l}\text { Growth } \\
(\mathrm{mg} / \mathrm{ml})\end{array}$ & $\begin{array}{c}\text { Antibiotics } \\
(\mu \mathrm{g} / \mathrm{ml})\end{array}$ \\
\hline 0.0 & 7.8 & 5.96 & $<10$ & 0.0 & 7.6 & 7.64 & $<10$ \\
\hline 0.05 & 5.4 & 11.0 & 22 & 0.05 & 6.0 & 8.51 & 24 \\
\hline 0.1 & 5.3 & 10.2 & 22 & 0.1 & 5.9 & 8.52 & 32 \\
\hline 0.2 & 5.1 & 11.1 & 23 & 0.2 & 6.0 & 11.3 & 20 \\
\hline 0.4 & 5.1 & 11.9 & 21 & 0.4 & 7.1 & 12.8 & $<10$ \\
\hline 1.0 & 7.0 & 8.6 & $<10$ & 1.0 & 7.1 & 11.2 & $<10$ \\
\hline $\begin{array}{c}\mathrm{CaCO}_{3} \\
(\%)\end{array}$ & $\mathrm{pH}$ & $\begin{array}{l}\text { Growth } \\
(\mathrm{mg} / \mathrm{ml})\end{array}$ & $\begin{array}{l}\text { Antibiotics } \\
(\mu \mathrm{g} / \mathrm{ml})\end{array}$ & $\underset{(\%)}{\mathrm{NaCl}}$ & $\mathrm{pH}$ & $\begin{array}{l}\text { Growth } \\
(\mathrm{mg} / \mathrm{ml})\end{array}$ & $\begin{array}{c}\text { Antibiotics } \\
(\mu \mathrm{g} / \mathrm{ml})\end{array}$ \\
\hline 0.0 & 5.2 & 5.94 & $<10$ & 0.0 & 5.2 & 11.0 & 24 \\
\hline 0.05 & 5.6 & 5.95 & 20 & 0.05 & 5.1 & 11.1 & 27 \\
\hline 0.1 & 5.1 & 13.6 & 25 & 0.1 & 5.1 & 11.9 & 28 \\
\hline 0.2 & 6.9 & 15.2 & 19 & 0.2 & 5.4 & 11.0 & 27 \\
\hline 0.4 & 7.2 & 12.7 & 15 & 0.4 & 5.4 & 11.9 & 24 \\
\hline 1.0 & 7.4 & 12.7 & $<10$ & 1.0 & 7.3 & 11.0 & 11 \\
\hline
\end{tabular}

Basal fermentation medium: soluble starch $4 \%$, mannitol $0.05 \%, \mathrm{NH}_{4} \mathrm{Cl} 0.5 \%$, L-arginine $0.5 \%, \mathrm{~K}_{2} \mathrm{HPO}_{4}$ $0.05 \%, \mathrm{MgSO}_{4} \cdot 7 \mathrm{H}_{2} \mathrm{O} 0.05 \%, \mathrm{NaCl} 0.1 \%, \mathrm{CaCO}_{3} 0.1 \%$.

Antibiotics were assayed by the agar diffusion assay using B. subtilis KY 4273 .

Table 5. Effects of trace metal mixtures

\begin{tabular}{c|c|c|c|c}
\hline $\begin{array}{c}\text { Trace metal } \\
\text { mixtures }\end{array}$ & $\begin{array}{c}\text { Concentration } \\
(\%)\end{array}$ & $\mathrm{pH}$ & $\begin{array}{c}\text { Growth } \\
(\mathrm{mg} / \mathrm{ml})\end{array}$ & $\begin{array}{c}\text { Fortimicins } \\
(\mu \mathrm{g} / \mathrm{ml})\end{array}$ \\
\hline none & & 4.9 & 17 & $<20$ \\
\hline & $1 \times 10^{-7}$ & 5.0 & 8 & 38 \\
$\mathrm{Fe}^{++}, \mathrm{Fe}^{+++}, \mathrm{Mo}^{++}$, & $1 \times 10^{-6}$ & 5.0 & 16 & 58 \\
$\mathrm{Co}^{++}, \mathrm{Ni}^{++}$ & $1 \times 10^{-5}$ & 5.0 & 15 & 62 \\
& $1 \times 10^{-4}$ & 5.1 & 3 & $<20$ \\
\hline $\mathrm{Mn}^{++}, \mathrm{Cr}^{+++}, \mathrm{Cu}^{++}$, & $1 \times 10^{-3}$ & 6.5 & 15 & $<20$ \\
$\mathrm{Zn}^{++}$ & $1 \times 10^{-7}$ & 4.9 & 15 & 29 \\
& $1 \times 10^{-5}$ & 5.3 & 9 & $<20$ \\
& $1 \times 10^{-4}$ & 6.6 & 3 & $<20$ \\
\hline
\end{tabular}

1) Each ions was added as the following salts
$\mathrm{Fe}^{++}: \quad \mathrm{FeSO}_{4} \cdot 7 \mathrm{H}_{2} \mathrm{O}$
$\mathrm{Co}^{++}: \mathrm{CoCl}_{2} \cdot 6 \mathrm{H}_{2} \mathrm{O}$
$\mathrm{Cr}^{+++}: \mathrm{Cr}_{2}\left(\mathrm{SO}_{4}\right)_{3} \cdot \mathrm{nH}_{2} \mathrm{O}$
$\mathrm{Fe}^{+++}: \mathrm{Fe}_{2}\left(\mathrm{SO}_{4}\right)_{3} \cdot \mathrm{nH}_{2} \mathrm{O}$
$\mathrm{Ni}^{++}: \mathrm{NiCl}_{2} \cdot 6 \mathrm{H}_{2} \mathrm{O}$
$\mathrm{Cu}^{++}: \mathrm{CuSO}_{4} \cdot 5 \mathrm{H}_{2} \mathrm{O}$
$\mathrm{Mo}^{++}: \quad\left(\mathrm{NH}_{4}\right)_{6} \mathrm{Mo}_{7} \mathrm{O}_{24} \cdot 4 \mathrm{H}_{2} \mathrm{O}$
$\mathrm{Mn}^{++}: \mathrm{MnSO}_{4} \cdot 4 \mathrm{H}_{2} \mathrm{O}$
$\mathrm{Zn}^{++}: \mathrm{ZnSO}_{4} \cdot 7 \mathrm{H}_{2} \mathrm{O}$

2) Final concentration of each ions in a medium

did not decrease as suddenly as in the control medium.

\section{Effects of Inorganic Salts}

The effects of such components as $\mathrm{K}_{2} \mathrm{HPO}_{4}, \mathrm{MgSO}_{4} \cdot 7 \mathrm{H}_{2} \mathrm{O}, \mathrm{NaCl}$ and $\mathrm{CaCO}_{3}$ were also examined (Table 4). All of them showed stimulatory effects both on the growth and antibiotic production at an optimum concentration of around $0.1 \%$. Though the effect of $\mathrm{NaCl}$ on the growth and maximum anti- 
Table 6. Single addition of trace metals

\begin{tabular}{|c|c|c|c|c|}
\hline $\begin{array}{l}\text { Trace } \\
\text { metals }\end{array}$ & $\begin{array}{c}\text { Concen- } \\
\text { tration } \\
(\%)\end{array}$ & $\mathrm{pH}$ & $\begin{array}{l}\text { Growth } \\
(\mathrm{mg} / \mathrm{ml})\end{array}$ & $\begin{array}{c}\text { Forti- } \\
\text { micins } \\
(\mu \mathrm{g} / \mathrm{ml})\end{array}$ \\
\hline none & & 4.5 & 16 & $<20$ \\
\hline \multirow[t]{3}{*}{$\mathrm{Fe}^{++}$} & $1 \times 10^{-5}$ & 6.9 & 11 & $<20$ \\
\hline & $1 \times 10^{-4}$ & 4.7 & 13 & $<20$ \\
\hline & $1 \times 10^{-3}$ & 5.9 & 15 & $<20$ \\
\hline \multirow{3}{*}{$\mathrm{Fe}^{+++}$} & $1 \times 10^{-5}$ & 5.3 & 11 & 24 \\
\hline & $1 \times 10^{-4}$ & 5.4 & 10 & 23 \\
\hline & $1 \times 10^{-3}$ & 5.4 & 11 & 24 \\
\hline \multirow{3}{*}{$\mathrm{Co}^{++}$} & $1 \times 10^{-5}$ & 5.1 & 10 & 62 \\
\hline & $1 \times 10^{-4}$ & 5.5 & 10 & 86 \\
\hline & $1 \times 10^{-3}$ & 6.0 & 6 & 26 \\
\hline \multirow{3}{*}{$\mathrm{Ni}^{++}$} & $1 \times 10^{-5}$ & 6.2 & 12 & 44 \\
\hline & $1 \times 10^{-4}$ & 6.3 & 10 & 75 \\
\hline & $1 \times 10^{-3}$ & 6.6 & 10 & 40 \\
\hline \multirow{3}{*}{$\mathrm{Mo}^{++}$} & $1 \times 10^{-5}$ & 5.3 & 11 & 30 \\
\hline & $1 \times 10^{-4}$ & 5.3 & 9 & 30 \\
\hline & $1 \times 10^{-3}$ & 5.4 & 13 & 24 \\
\hline $\begin{array}{l}\mathrm{Fe}^{++} \\
\mathrm{Fe}^{+++} \\
\mathrm{Mo}^{++} \\
\mathrm{Co}^{++} \\
\mathrm{Ni}^{++}\end{array}$ & $1 \times 10^{-4}$ & 5.1 & 13 & 95 \\
\hline
\end{tabular}

Table 7. Effects of vitamin mixtures

\begin{tabular}{l|c|c|c|c}
\hline $\begin{array}{c}\text { Vitamin } \\
\text { mixtures }^{1)}\end{array}$ & $\begin{array}{c}\text { Concen- } \\
\text { tration } \\
(\%)\end{array}$ & $\mathrm{pH}$ & $\begin{array}{c}\text { Growth } \\
(\mathrm{mg} / \mathrm{ml})\end{array}$ & $\begin{array}{c}\text { Forti- } \\
\text { micins } \\
(\mu \mathrm{g} / \mathrm{ml})\end{array}$ \\
\hline none & & 5.3 & 14 & 30 \\
\hline & $1 \times 10^{-7}$ & 7.6 & 4 & $<16$ \\
& $1 \times 10^{-6}$ & 5.3 & 11 & 42 \\
{$[\mathrm{~A}]$} & $1 \times 10^{-5}$ & 5.4 & 12 & 72 \\
& $1 \times 10^{-4}$ & 5.4 & 13 & 80 \\
& $1 \times 10^{-3}$ & 7.6 & 5 & $<16$ \\
\hline & $1 \times 10^{-7}$ & 5.6 & 18 & 22 \\
& $1 \times 10^{-6}$ & 6.1 & 13 & 17 \\
{$[\mathrm{~B}]$} & $1 \times 10^{-5}$ & 5.5 & 10 & 28 \\
& $1 \times 10^{-4}$ & 5.8 & 15 & 26 \\
& $1 \times 10^{-3}$ & 6.7 & 12 & $<16$ \\
\hline
\end{tabular}

1) [A] pyridoxine, pyridoxal, niacin, biotin, vitamin $\mathrm{B}_{12}$

[B] Ca-pantothenate, riboflavin, thiamine, p-aminobenzoic acid, folic acid

2) Final concentrations of each vitamin in a medium

biotic production was not clear, it was effective in stabilizing the fermentation. With $0.1 \%$ of $\mathrm{NaCl}$, the maximum antibiotic yield continued

from the 4th to 6 th day, while under other conditions it did not.

Effects of Trace Metals

Trace metals were tested in two groups: (1) $\mathrm{Fe}^{++}, \mathrm{Fe}^{+++}, \mathrm{Mo}^{++}, \mathrm{Co}^{++}$and $\mathrm{Ni}^{++}$, and (2) $\mathrm{Mn}^{++}$, $\mathrm{Cr}^{+++}, \mathrm{Cu}^{++}$and $\mathrm{Zn}^{++}$. The mixtures were added to a fermentation medium containing $4 \%$ soluble starch, $0.05 \%$ mannitol, $0.5 \% \mathrm{NH}_{4} \mathrm{Cl}, 0.5 \%$ L-arginine, $0.05 \% \mathrm{~K}_{2} \mathrm{HPO}_{4}, 0.1 \% \mathrm{MgSO}_{4} \cdot 7 \mathrm{H}_{2} \mathrm{O}, 0.1 \%$ $\mathrm{NaCl}$ and $0.1 \% \mathrm{CaCO}_{3}$, at concentrations of $1 \times 10^{-7} \%$ to $1 \times 10^{-3} \%$ as each ion. As shown in Table 5 , group 1 exhibited a significant effect on the antibiotic production at a concentration of $1 \times 10^{-4} \%$,

Table 8. Effect of vitamin $\mathrm{B}_{12}$

\begin{tabular}{c|c|c|c|c|c}
\hline \multirow{2}{*}{$\begin{array}{c}\text { Vitamin } \\
(\%)\end{array}$} & $\mathrm{pH}$ & $\begin{array}{c}\text { Growth } \\
(\mathrm{mg} / \mathrm{ml})\end{array}$ & $\begin{array}{c}\text { Antibiotics }(\mu \mathrm{g} / \mathrm{ml}) \\
\text { fortimicins }\end{array}$ & $\begin{array}{c}\text { Fortimicin } \\
\mathrm{A}\end{array}$ & $\begin{array}{c}\text { Fortimicin } \\
\mathrm{B}\end{array}$ \\
\hline none & 5.5 & 10 & 20 & 19 & $\operatorname{tr}$ \\
\hline $1 \times 10^{-5}$ & 5.6 & 9 & 40 & 32 & $\operatorname{tr}$ \\
$2 \times 10^{-5}$ & 5.3 & 9 & 40 & 30 & $\operatorname{tr}$ \\
$5 \times 10^{-5}$ & 6.4 & 12 & 48 & 32 & $\operatorname{tr}$ \\
$1 \times 10^{-4}$ & 4.8 & 10 & 54 & 45 & $\operatorname{tr}$ \\
$2 \times 10^{-4}$ & 5.2 & 12 & 33 & 32 & $\operatorname{tr}$ \\
$5 \times 10^{-4}$ & 5.0 & 10 & 36 & 37 & $\operatorname{tr}$ \\
$1 \times 10^{-3}$ & 5.4 & 11 & 40 & 35 & \\
\hline
\end{tabular}


Table 9. Simultaneous addition of vitamin $\mathrm{B}_{12}$ and $\mathrm{Co}^{++}$

\begin{tabular}{|c|c|c|c|c|c|c|}
\hline \multirow{2}{*}{$\begin{array}{l}\mathrm{Co}^{++} \\
(\%)\end{array}$} & \multirow{2}{*}{$\underset{(\%)}{\operatorname{Vitamin}} \mathrm{B}_{12}$} & \multirow{2}{*}{$\mathrm{pH}$} & \multirow{2}{*}{$\begin{array}{l}\text { Growth } \\
(\mathrm{mg} / \mathrm{ml})\end{array}$} & \multicolumn{3}{|c|}{ Antibiotics $(\mu \mathrm{g} / \mathrm{ml})$} \\
\hline & & & & $\begin{array}{c}\text { Total } \\
\text { fortimicins }\end{array}$ & $\begin{array}{c}\text { Fortimicin } \\
\text { A }\end{array}$ & $\begin{array}{c}\text { Fortimicin } \\
\text { B }\end{array}$ \\
\hline $1 \times 10^{-5}$ & - & 5.1 & 9 & 60 & 48 & $\operatorname{tr}$ \\
\hline $1 \times 10^{-4}$ & - & 5.4 & 8 & 74 & 69 & $\operatorname{tr}$ \\
\hline $1 \times 10^{-3}$ & - & 7.7 & 7 & 30 & 25 & $\operatorname{tr}$ \\
\hline - & $1 \times 10^{-5}$ & 5.6 & 9 & 40 & 31 & $\operatorname{tr}$ \\
\hline- & $1 \times 10^{-4}$ & 5.0 & 9 & 57 & 46 & $\operatorname{tr}$ \\
\hline - & $1 \times 10^{-3}$ & 5.3 & 10 & 39 & 35 & $\operatorname{tr}$ \\
\hline $1 \times 10^{-4}$ & $1 \times 10^{-5}$ & 5.4 & 10 & 64 & 52 & $\operatorname{tr}$ \\
\hline $1 \times 10^{-4}$ & $1 \times 10^{-4}$ & 5.0 & 10 & 70 & 68 & $\operatorname{tr}$ \\
\hline $1 \times 10^{-4}$ & $1 \times 10^{-3}$ & 5.3 & 8 & 58 & 43 & $\operatorname{tr}$ \\
\hline- & - & 5.5 & 10 & 20 & 16 & $\operatorname{tr}$ \\
\hline
\end{tabular}

although the growth was slightly inhibited. The maximum total potency was $90 \mu \mathrm{g} / \mathrm{ml}$ as total fortimicins while the no addition control was $20 \mu \mathrm{g} / \mathrm{ml}$. Group 2 showed no effect on antibiotic production.

$\mathrm{Co}^{++}$and $\mathrm{Ni}^{++}$were subsequently selected as effective metals by single omission and single addition tests (Table 6). In Tables 5 and 6, the increase in total antibiotic production were mainly due to the increase of fortimicin A, with only a slight effect on the production of fortimicin B.

The slight stimulatory effect of simultaneous addition of nickel with cobalt was also evident. As shown in Fig. 3, when $1 \times 10^{-4} \%$ of $\mathrm{Ni}^{++}$was added to the cobalt-containing fermentation medium, the total potency and fortimicin A formation were higher than cobalt-containing control condition on the 4th day, but the effect of nickel disappeared finally. On the 6th day the total potency and fortimicin A in a nickel-containing condition were the same as those of the control; pH's of flasks containing nickel were also higher from 3 to 6 days. The production of fortimicin $\mathrm{B}$ was almost the same for the two conditions.

\section{Effects of Vitamins}

A mixture [A] of pyridoxine, pyridoxal, niacin, biotin and vitamin $\mathrm{B}_{12}$, and another mixture [B] containing calcium-pantothenate, riboflavin, thiamine, $p$-aminobenzoic acid and folic acid were added to a basal fermentation medium containing $4 \%$ soluble starch, $0.05 \%$ mannitol, $0.5 \% \mathrm{NH}_{4} \mathrm{Cl}, 0.5 \%$ L-arginine, $0.05 \% \mathrm{~K}_{2} \mathrm{HPO}_{4}, 0.1 \% \mathrm{MgSO}_{4} \cdot 7 \mathrm{H}_{2} \mathrm{O}, 0.1 \% \mathrm{NaCl}$ and $0.1 \% \mathrm{CaCO}_{3}$, at concentrations of $1 \times 10^{-7} \%$ to $1 \times 10^{-3} \%$ of each vitamin. The first mixture [A] gave two to three times higher total antibiotic potency than the control, while the second mixture [B] showed no effect on the potency (Table 7). By single omission and single addition tests in the first mixture $[\mathrm{A}]$, it was shown that vitamin $\mathrm{B}_{12}$ could be substituted for the mixture. As shown in Table 8, vitamin $\mathrm{B}_{12}$ was found effective for stimulating the total potency by bioassay which was mainly due to the increase of fortimicin A without any effect on the growth. Vitamin $\mathbf{B}_{12}$ had much less effect on the fortimicin B production. The effect of simultaneous addition of vitamin $\mathrm{B}_{12}$ with cobalt was almost the same as that with cobalt alone (Table 9).

\section{Discussion}

Among various kinds of inorganic nitrogen compounds, ammonium ion improved growth more 
than nitrate. This organism was not able to utilize nitrite. The results shown in Table 1 agree with those reported for Micromonospora purpurea by WAGMAN et al. ${ }^{8)}$ with the exception of $\left(\mathrm{NH}_{4}\right)_{2} \mathrm{SO}_{4}$. They reported that $M$. purpurea could not utilize $\left(\mathrm{NH}_{4}\right)_{2} \mathrm{SO}_{4} . M$. olivoasterospora however could utilize the compound as well as $\mathrm{NH}_{4} \mathrm{NO}_{3}$ or $\mathrm{NH}_{4} \mathrm{Cl}$. Many other compounds which improve the growth of $M$. olivoasterospora agree with those of $M$. purpurea. $\mathrm{K}_{2} \mathrm{HPO}_{4}, \mathrm{MgSO}_{4}$ and $\mathrm{CaCO}_{3}$ showed some effect on the growth of $M$. olivoasterospora (Table 4). $\mathrm{NaHCO}_{3}$ and $\mathrm{Na}_{2} \mathrm{CO}_{3}$ could be substituted for $\mathrm{CaCO}_{3}$, although the growth in both instances was not so good as with $\mathrm{CaCO}_{3} . \mathrm{CaCl}_{2}$ resulted in no growth. The effective carbon sources of soluble starch, glucose, maltose and sucrose, and the effective amino acids of L-asparagine, L-citrulline, L-arginine and L-glutamic acid were the same as in the case of $M$. purpurea. Both organisms thus show almost the same nutritional requirements for growth, although some differences were noted.

The addition of $\mathrm{Ni}^{++}$at the concentration of $1 \times 10^{-4} \%$ to the fermentation medium without cobalt showed better antibiotic production than the control (Table 6). Initially it was felt that the effect of nickel, might be due to cobalt contaminating the nickel, as in the case of coumermycin A. ${ }^{10)}$ The nickel chloride used in the experiments contained, however, only $0.049 \%$ of $\mathrm{Co}^{++}$. The effect of nickel could be hardly explained by contamination by cobalt in the nickel chloride. $1 \times 10^{-4} \%$ of $\mathrm{Ni}^{++}$added with $1 \times 10^{-4} \%$ of $\mathrm{Co}^{++}$showed better effect on both total antibiotic production and fortimicin A production in the earlier fermentation phase (around 4 days, Fig. 3), although the effect on fortimicin B was far less than on fortimicin A.

Fortimicin $\mathrm{A}$ in phosphate buffer of alkaline $\mathrm{pH}$ is unstable, and degrades easily to fortimicin $\mathrm{B}$ at the temperature around $30^{\circ} \mathrm{C}$, although degradation of fortimicin $\mathrm{B}$ is very small. Degradation increases as the $\mathrm{pH}$ rises, and is more rapid as the temperature increases. Therefore, it is very likely that accumulation of fortimicin $\mathrm{B}$ in the later phase of fermentation carried out at $30^{\circ} \mathrm{C}$, is due to the chemical degradation of fortimicin A produced in the earlier phase of fermentation. Mannitol was found effective to keep $\mathrm{pH}$ of the broth lower than the control. The total potency of the broth with mannitol might be higher than the control then, by preventing the degradation of fortimicin A. Although glucose was more effective in keeping the $\mathrm{pH}$ of broth lower than mannitol and, of course than the control, it actually decreased the total antibiotic potency slightly.

Vitamin $\mathrm{B}_{12}$ also showed a stimulatory effect on antibiotic production (Table 8 ). The effect was however hidden thoroughly by cobalt added simultaneously with it (Table 9). Cobalt must contribute to formation of vitamin $\mathbf{B}_{12}$ by $M$. olivoasterospora as reported in other species of the genus Micromonospora ${ }^{11}$. A part of the effects of cobalt can be thus ascribed to that of vitamin $\mathrm{B}_{12}$. As the effect of cobalt on antibiotic production is however greater than that of vitamin $\mathbf{B}_{12}$, as shown in Table 9 , the former may be involved in another mechanism for the antibiotic production besides formation of the latter.

Tilley et al. reported the involvement of cobalt in methylation for gentamicin fermentation with a mutant of $M$. purpurea. $^{12)}$

Effect of L-methionine as a possibility was then examined. However, L-methionine could not be substituted for cobalt or vitamin $\mathrm{B}_{12}$.

The bioassay value for total antibiotics was usually higher than the chemical assay value for fortimicin A, as the case of Fig. 3. As fortimicin B has almost no antibiotic activity against B. subtilis, the test organism, the difference between the two assay methods may suggest the existence of some other active components against $B$. subtilis in the broth besides fortimicins A and B.

\section{Acknowledgements}

We express our appreciation to Mr. J. Theriault, Dr. A. Sinclair, Miss M. Jackson and associates in Abbott Labs. for their kind advice and encouragement.

\section{References}

1) Nara, T.; M. Yamamoto, I. Kawamoto, K. Takayama, R. OKachi, S. Takasawa, T. Sato \& S. Sato: 
Fortimicins A and B, new aminoglycoside antibiotics. I. Producing organism, fermentation and biological properties of fortimicins. J. Antibiotics 30: 533 540, 1977

2) OKachi, R.; S. Takasawa, T. Sato, S. Sato, M. Yamamoto, I. Kawamoto \& T. Nara: Fortimicins A and $\mathrm{B}$, new aminoglycoside antibiotics. II. Isolation, physico-chemical and chromatographic properties. J. Antibiotics 30: 541 551, 1977

3) Kawamoto, I.; M. Yамамото \& T. Nara: Producing organism of fortimicins. Abst. papers No. 2D-26, Annual Meeting of the Agricultural Chemical Society of Japan, Tokyo, p. 258, April 1 4, 1977

4) Kawamoto, I.; T. OKa \& T. NaRA: Physiological properties of a fortimicin-producing Micromonospora olivoasterospora. Abstracts, No. 4, Annual Meeting of the Actinomycetologist, Tokyo, May 20, 1977

5) Egan, R. S.; R. S. Stanaszek, M. Cirovic, S. L. Mueller, J. Tadanier, J. R. Martin, P. Collum, A. W. Goldstein, R. L. DeVault, A. C. Sinclatr, E. E. Fager \& L. A. Mitscher: Fortimicins A and B, new aminoglycoside antibiotics. III. Structural identification. J. Antibiotics 30: 552 563, 1977

6) Girolami, R. L. \& J. M. Stamm: Fortimicins A and B, new aminoglycoside antibiotics. IV. In vitro study of fortimicin A compared with other aminoglycosides. J. Antibiotics 30: 564 570, 1977

7) Testa, R. T. \& L. Kamnitzer: Gentamicin production in a synthetic medium. Abst. papers No. E-107, p. 18, 47th Annual Meeting of Am. Soc. Microbiol., Chicago, May 12 17, 1974

8) Wagman, G. H. \& M. J. Weinstein: A chemically defined fermentation medium for the growth of Micromonospora purpurea. Biotech. Bioeng. 8: 259 273, 1966

9) Benjamin, D. M.; J. J. McCormack \& D. W. Gump: Use of newer amino group reagents for the detection and determination of kanamycin. Anal. Chem. 45: 1531 1534, 1973

10) Claridge, C. A.; V. Z. Rossomano, N. S. Buono, A. Gourevitch \& J. Lein: Influence of cobalt on fermentative methylation. Appl. Microbiol. 14: 280 283, 1966

11) Wagman, G. H.; R. D. Gannon \& M. J. Weinstein: Production of vitamin $\mathrm{B}_{12}$ by Micromonospora. Appl. Microbiol. 17: 648 649, 1969

12) Tilley, B. C.; R. T. Testa \& E. Dorman: A role of cobalt ions in the biosynthesis of gentamicin. Abst. papers of 31st Meeting for Soc. Indust. Microbiol., Aug. 17 22, 1975 